\title{
Partial Element Equivalent Circuit Models in the Solution of the Electric Field Integral Equation
}

\author{
Francesco Ferranti* Giulio Antonini ${ }^{\dagger} \quad$ Krishnan Chemmangat* \\ Luc Knockaert* Tom Dhaene*
}

\begin{abstract}
D electromagnetic methods are fundamental design tools for complex high-speed systems. Among the integral equation-based techniques, the Partial Element Equivalent Circuit (PEEC) method has received a special attention in interconnect modeling, where mixed electromagnetic/circuit problems need to be solved. Retardation effects and the resulting delays must be taken into account and included in the modeling, when signal waveform rise times decrease and the corresponding frequency content increases or the geometric dimensions become electrically long. In this case, the enforcement of the Kirchhoff laws to PEEC delayed models leads to a set of delayed differential equations in a neutral form. The aim of this contribution is to present an overview of the PEEC method with special focus on the analysis of electrically long structures that require taking delays into account.
\end{abstract}

\section{Introduction}

The increasing demand for performance of integrated circuits (ICs) pushes operation to higher signal bandwidths and accurate modeling of previously neglected second order effects, such as crosstalk, reflection, delay and coupling, becomes increasingly important during circuit and system simulations $[1,2]$. Electromagnetic (EM) 3-D methods $[3,4,5]$ have become increasingly indispensable analysis and design tools for a variety of complex high-speed systems. Among all EM methods, the Partial Element Equivalent Circuit (PEEC) method [4] has been found particularly useful for modeling PCBs and interconnects. The PEEC method uses a circuit interpretation of the Electric Field Integral Equation (EFIE) [6], thus being especially suitable to problems involving both electromagnetic fields and circuits $[2,4,7]$. Nonlinear circuit devices such as drivers and receivers are usually connected to PEEC equivalent circuits using a time domain circuit simulator (e.g. SPICE [8]). The quasi-static PEEC formulation [4], which approximates the full-wave PEEC approach [9],

\footnotetext{
*Department of Information Technology, Ghent University - IBBT, Gaston Crommenlaan 8 Bus 201, B-9050, Gent, Belgium., e-mail: \{francesco.ferranti, krishnan.cmc, luc.knockaert, tom.dhaene\}@intec.ugent.be.

${ }^{\dagger}$ UAq EMC Laboratory, Dipartimento di Ingegneria Elettrica e dell'Informazione, Università degli Studi dell'Aquila, Via G. Gronchi 18, 67100, L'Aquila, Italy, email: giulio.antonini@univaq.it.
}

yields an equivalent RLC circuit by neglecting the time delays between the elements in the full-wave PEEC formulation. Systems of ordinary differential equations (ODE) with constant coefficients in the time-domain and complex algebraic systems of equations with frequency-independent matrices in the frequency domain are obtained. The quasistatic PEEC formulation is reasonable for problems with electrically small geometries. When signal waveform rise times decrease and the corresponding frequency content increases or the geometric dimensions become electrically large, time delays must be taken into account and included in the modeling.

A PEEC formulation which includes delay elements, called $\tau$ PEEC method [10], becomes necessary and leads to systems of neutral delayed differential equations (NDDE) [9] with constant coefficients and constant delay times in the time domain and to complex algebraic systems of equations with frequency-dependent matrices in the frequency domain. Simply using quasi-static PEEC models can result in significant errors and artifacts in the modeling [11].

This paper presents an overview of the PEEC method in the solution of the EFIE with special focus on the analysis of electrically long structures that require taking delays into account.

\section{Delayed PEEC formulation}

The PEEC method [4] stems from the integral equation form of Maxwell's equations.

The main difference of the PEEC method with other integral equation based techniques such as the Method of Moments [3] resides in the fact that it provides a circuit interpretation of the EFIE [6] in terms of partial elements, namely resistances, partial inductances and coefficients of potential. Thus, the resulting equivalent circuit can be studied by means of SPICE-like circuit solvers [8] in both time and frequency domain.

The PEEC method is based on the EFIE [6] of the form

$$
\boldsymbol{E}^{i}=\frac{\boldsymbol{J}(\boldsymbol{r}, t)}{\sigma}+\frac{\partial \boldsymbol{A}(\boldsymbol{r}, t)}{\partial t}+\nabla \phi(\boldsymbol{r}, t)
$$

where $\boldsymbol{E}^{i}$ is an incident electric field, $\boldsymbol{J}$ is a current 
density, $\boldsymbol{A}$ is the vector magnetic potential, and $\phi$ is the scalar electric potential at observation point $\boldsymbol{r}$. The standard Galerkin-type approach $[3,12]$ is applied by expanding current and charge densities using pulse basis functions. Scalar and vector potentials, $\phi(\boldsymbol{r}, t)$ and $\boldsymbol{A}(\boldsymbol{r}, t)$ respectively, are analogously expanded. By defining a specific inner product, as weighted volume integral over an elementary volume, (1) can be interpreted as Kirchhoff Voltage Law (KVL). The discretization process leads to topological entities such as nodes and branches, thus making the continuous electromagnetic problem solvable as an equivalent circuit [4]. In addition, the continuity equation is to be enforced

$$
\nabla \cdot \boldsymbol{J}(\boldsymbol{r}, j \omega)+j \omega \rho(\boldsymbol{r}, j \omega)=0
$$

which can be easily converted in an equivalent circuit form leading to the Kirchhoff Current Law (KCL). The charge density is represented by $\rho(\boldsymbol{r}, j \omega)$. The resulting equivalent circuit can be studied by means of SPICE-like circuit solvers [8] in both time- and frequency-domain.

Following the standard approach [4], volumes and surfaces are discretized into elementary regions, hexahedra and patches respectively [10]. The current and charge densities are expanded into a series of basis functions which are usually assumed as pulse basis functions. The choice of pulse basis functions implies to assume constant current and charge densities over the elementary volume (inductive) and surface (capacitive) cells, respectively.

Conductors are modeled by their ohmic resistance, dielectrics by the excess capacitance [13]. Hence, magnetic and electric field coupling are modeled by partial inductances and coefficients of potential, respectively.

The magnetic field coupling between two inductive volume cells $\alpha$ and $\beta$ is modeled by the partial inductance

$$
L p_{\alpha \beta}=\frac{\mu}{4 \pi} \frac{1}{a_{\alpha} a_{\beta}} \int_{u_{\alpha}} \int_{u_{\beta}} \frac{1}{R_{\alpha \beta}} d u_{\alpha} d u_{\beta}
$$

where $R_{\alpha \beta}$ is the distance between any two points in volumes $u_{\alpha}$ and $u_{\beta}$ with $a_{\alpha}$ and $a_{\beta}$ their cross sections. The electric field coupling between two capacitive surface cells $\gamma$ and $\delta$ is modeled by the coefficient of potential

$$
P_{\gamma \delta}=\frac{1}{4 \pi \varepsilon} \frac{1}{S_{\gamma} S_{\delta}} \int_{S_{\gamma}} \int_{S_{\delta}} \frac{1}{R_{\gamma \delta}} d S_{\gamma} d S_{\delta}
$$

where $R_{\gamma \delta}$ is the distance between any two points on surfaces $\gamma$ and $\delta$, while $S_{\gamma}$ and $S_{\delta}$ denote the area of their respective surfaces.
Due to the finite value of the speed of light, partial inductances and coefficients of potentials relate causes and effects delayed in time:

$$
\begin{aligned}
& v_{L, \alpha}(t)=L p_{\alpha \beta} \frac{d i_{\beta}\left(t-\tau_{\alpha \beta}\right)}{d t} \\
& v_{P, \gamma}(t)=P_{\gamma \delta} q_{\delta}\left(t-\tau_{\gamma \delta}\right)
\end{aligned}
$$

where $\tau_{\alpha \beta}=R_{\alpha \beta} / c_{0}$ and $\tau_{\gamma \delta}=R_{\gamma \delta} / c_{0}$ are the center-to-center delays between the corresponding basis-function domains, and $c_{0}$ denotes the freespace speed of light. Hence, partial inductance and coefficient of potential matrices act as a delay operator for time derivatives of currents and charges, respectively

$$
\begin{aligned}
\mathbf{v}_{L}(t) & =\mathbf{L}_{p} \frac{d \mathbf{i}\left(t-\boldsymbol{\tau}_{L}\right)}{d t}=\widetilde{\mathbf{L}}_{p}\left(\boldsymbol{\tau}_{L}\right) \frac{d \mathbf{i}(t)}{d t} \\
\mathbf{v}(t) & =\mathbf{P} \mathbf{q}\left(t-\boldsymbol{\tau}_{C}\right)=\widetilde{\mathbf{P}}\left(\boldsymbol{\tau}_{C}\right) \mathbf{q}(t)
\end{aligned}
$$

where $\boldsymbol{\tau}_{L}$ and $\boldsymbol{\tau}_{C}$ denote the center-to-center delay matrices for the magnetic and electric field couplings, respectively.

Generalized Kirchoff's laws, for conductors, can be rewritten as

$$
\begin{aligned}
& \frac{d \mathbf{q}(t)}{d t}-\mathbf{A}^{T} \mathbf{i}(t)+\mathbf{i}_{e}(t)=0 \\
& -\mathbf{A v}(t)-\widetilde{\mathbf{L}}_{p}\left(\boldsymbol{\tau}_{L}\right) \frac{d \mathbf{i}(t)}{d t}-\mathbf{R i}(t)=\mathbf{0}
\end{aligned}
$$

where $\mathbf{A}$ is the connectivity matrix, $\mathbf{v}(t)$ denotes the node potentials to infinity, $\mathbf{i}(t)$ and $\mathbf{i}_{e}(t)$ represent the currents flowing in volume cells and the external currents, respectively.

The previous equation ( $8 \mathrm{~b}$ ) has to be modified when dielectrics are considered, since the resistance $\mathbf{R}$ is substituted by the excess capacitance which relates the polarization charge and the corresponding voltage drop as $\mathbf{v}_{d}(t)=\mathbf{C}_{d}^{-1} \mathbf{q}_{d}(t)$ [13]. Hence, for dielectric elementary cells, (8) become

$$
\begin{aligned}
& \frac{d \mathbf{q}(t)}{d t}-\mathbf{A}^{T} \mathbf{i}(t)+\mathbf{i}_{e}(t)=0 \\
& -\mathbf{A} \mathbf{v}(t)-\widetilde{\mathbf{L}}_{p}\left(\boldsymbol{\tau}_{L}\right) \frac{d \mathbf{i}(t)}{d t}-\mathbf{v}_{d}(t)=\mathbf{0} \\
& \mathbf{i}(t)=\mathbf{C}_{d} \frac{d \mathbf{v}_{d}(t)}{d t}
\end{aligned}
$$

A selection matrix $\mathbf{K}$ is introduced to define the port voltages by selecting node potentials. The same matrix is used to obtain the external currents $\mathbf{i}_{e}(t)$ by the currents $\mathbf{i}_{s}(t)$ which are of opposite sign with respect to the port currents $\mathbf{i}_{p}(t)$

$$
\begin{aligned}
\mathbf{v}_{p}(t) & =\mathbf{K} \mathbf{v}(t) \\
\mathbf{i}_{e}(t) & =\mathbf{K}^{T} \mathbf{i}_{s}(t)
\end{aligned}
$$


An example of $\tau$ PEEC circuit for a conductor elementary cell is illustrated, in the Laplace domain, in Fig. 1 where the current controlled voltage sources $s L_{p, i j} I_{j}$ and the charge controlled current sources $s Q_{i}$ model the magnetic and electric field couplings, respectively.

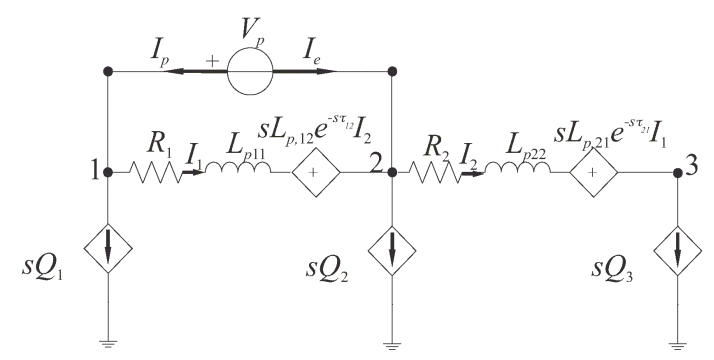

Figure 1: Illustration of $\tau$ PEEC circuit electrical quantities for a conductor elementary cell.

\section{Descriptor representation of $\tau$ PEEC cir- cuits}

We assume that the system under analysis consists of conductors and dielectrics. Let us denote with $n_{n}$ the number of nodes and $n_{i}$ the number of branches where currents flow. Among the latter, we denote with $n_{c}$ and $n_{d}$ the number of branches of conductors and dielectrics, respectively. Furthermore, let us assume to be interested in generating an admittance representation having $n_{p}$ output currents $\mathbf{i}_{p}(t)$ under voltage excitation $\mathbf{v}_{p}(t)$. Since dielectrics require the excess capacitance to model the polarization charge [13], additional $n_{d}$ unknowns are needed in addition to currents. Hence, if the MNA approach [14] is used, the global number of unknowns is $n_{u}=n_{i}+n_{d}+n_{n}+n_{p}$. In a matrix form, (8)-(10), taking (7) into account, read

$$
\begin{aligned}
& \underbrace{\left[\begin{array}{cccc}
\mathbf{I}_{n_{n}, n_{n}} & \mathbf{0}_{n_{n}, n_{i}} & \mathbf{0}_{n_{n}, n_{d}} & \mathbf{0}_{n_{n}, n_{p}} \\
\mathbf{0}_{n_{i}, n_{n}} & \widetilde{\mathbf{L}}_{p}\left(\boldsymbol{\tau}_{L}\right) & \mathbf{0}_{n_{i}, n_{d}} & \mathbf{0}_{n_{i}, n_{p}} \\
\mathbf{0}_{n_{d}, n_{n}} & \mathbf{0}_{n_{d}, n_{i}} & \mathbf{C}_{d} & \mathbf{0}_{n_{d}, n_{p}} \\
\mathbf{0}_{n_{p}, n_{n}} & \mathbf{0}_{n_{p}, n_{i}} & \mathbf{0}_{n_{p}, n_{d}} & \mathbf{0}_{n_{p}, n_{p}}
\end{array}\right]}_{\mathbf{C}} \cdot \frac{d}{d t} \mathbf{x}(t)= \\
& \underbrace{\left[\begin{array}{cccc}
\mathbf{0}_{n_{n}, n_{n}} & -\mathbf{A}^{T} & \mathbf{0}_{n_{n}, n_{d}} & \mathbf{K}^{T} \\
\mathbf{A} \widetilde{\mathbf{P}}\left(\boldsymbol{\tau}_{C}\right) & \mathbf{R} & \boldsymbol{\Phi} & \mathbf{0}_{n_{i}, n_{p}} \\
\mathbf{0}_{n_{d}, n_{n}} & -\boldsymbol{\Phi}^{T} & \mathbf{0}_{n_{d}, n_{d}} & \mathbf{0}_{n_{d}, n_{p}} \\
-\mathbf{K} \widetilde{\mathbf{P}}\left(\boldsymbol{\tau}_{C}\right) & \mathbf{0}_{n_{p}, n_{i}} & \mathbf{0}_{n_{p}, n_{d}} & \mathbf{0}_{n_{p}, n_{p}}
\end{array}\right]}_{\mathbf{G}} \cdot \mathbf{x}(t)+ \\
& \underbrace{\left[\begin{array}{c}
\mathbf{0}_{n_{n}+n_{i}+n_{d}, n_{p}} \\
-\mathbf{I}_{n_{p}, n_{p}}
\end{array}\right]}_{\mathbf{B}} \cdot \underbrace{\left[\mathbf{v}_{p}(t)\right]}_{\mathbf{u}(t)}
\end{aligned}
$$

where $\mathbf{x}(t)=\left[\begin{array}{llll}\mathbf{q}(t) & \mathbf{i}(t) & \mathbf{v}_{d}(t) & \mathbf{i}_{s}(t)\end{array}\right]^{T} \in \Re^{n_{u} \times 1}$ and $\mathbf{I}_{n_{p}, n_{p}}$ is the identity matrix of dimensions equal to the number of ports. Matrix $\boldsymbol{\Phi}$ is

$$
\boldsymbol{\Phi}=\left[\begin{array}{c}
\mathbf{0}_{n_{c}, n_{d}} \\
\mathbf{I}_{n_{d}, n_{d}}
\end{array}\right]
$$

If the delay operator $\widetilde{\mathbf{P}}\left(\boldsymbol{\tau}_{C}\right)$ is applied to the first equation in (11), the system can be recast as

$$
\begin{aligned}
& \underbrace{\left[\begin{array}{cccc}
\widetilde{\mathbf{P}}\left(\boldsymbol{\tau}_{C}\right) & \mathbf{0}_{n_{n}, n_{i}} & \mathbf{0}_{n_{n}, n_{d}} & \mathbf{0}_{n_{n}, n_{p}} \\
\mathbf{0}_{n_{i}, n_{n}} & \widetilde{\mathbf{L}}_{p}\left(\boldsymbol{\tau}_{L}\right) & \mathbf{0}_{n_{i}, n_{d}} & \mathbf{0}_{n_{i}, n_{p}} \\
\mathbf{0}_{n_{d}, n_{n}} & \mathbf{0}_{n_{d}, n_{i}} & \mathbf{C}_{d} & \mathbf{0}_{n_{d}, n_{p}} \\
\mathbf{0}_{n_{p}, n_{n}} & \mathbf{0}_{n_{p}, n_{i}} & \mathbf{0}_{n_{p}, n_{d}} & \mathbf{0}_{n_{p}, n_{p}}
\end{array}\right]}_{\mathbf{C}} \cdot \frac{d}{d t} \mathbf{x}(t)= \\
& \underbrace{\left[\begin{array}{cccc}
\mathbf{0}_{n_{n}, n_{n}} & -\widetilde{\mathbf{P}}\left(\boldsymbol{\tau}_{C}\right) \mathbf{A}^{T} & \mathbf{0}_{n_{n}, n_{d}} & \widetilde{\mathbf{P}}\left(\boldsymbol{\tau}_{C}\right) \mathbf{K}^{T} \\
\mathbf{A} \widetilde{\mathbf{P}}\left(\boldsymbol{\tau}_{C}\right) & \mathbf{R} & \boldsymbol{\Phi} & \mathbf{0}_{n_{i}, n_{p}} \\
\mathbf{0}_{n_{d}, n_{n}} & -\boldsymbol{\Phi}^{T} & \mathbf{0}_{n_{d}, n_{d}} & \mathbf{0}_{n_{d}, n_{p}} \\
-\mathbf{K} \widetilde{\mathbf{P}}\left(\boldsymbol{\tau}_{C}\right) & \mathbf{0}_{n_{p}, n_{i}} & \mathbf{0}_{n_{p}, n_{d}} & \mathbf{0}_{n_{p}, n_{p}}
\end{array}\right]}_{\mathbf{G}} . \\
& \cdot \mathbf{x}(t)+\underbrace{\left[\begin{array}{c}
\mathbf{0}_{n_{n}+n_{i}+n_{d}, n_{p}} \\
-\mathbf{I}_{n_{p}, n_{p}}
\end{array}\right]}_{\mathbf{B}} \cdot \underbrace{\left[\mathbf{v}_{p}(t)\right]}_{\mathbf{u}(t)}
\end{aligned}
$$

In a more compact form, the previous equations can be rewritten as

$$
\begin{aligned}
\mathbf{C}(\boldsymbol{\tau}) \frac{d \mathbf{x}(t)}{d t} & =-\mathbf{G}(\boldsymbol{\tau}) \mathbf{x}(t)+\mathbf{B u}(t) \\
\mathbf{i}_{p}(t) & =\mathbf{L}^{T} \mathbf{x}(t)
\end{aligned}
$$

where $\boldsymbol{\tau} \in \Re^{n_{\tau} \times 1}$ contains all delays $\boldsymbol{\tau}_{L}, \boldsymbol{\tau}_{C}$. Since this is an $n_{p}$-port formulation, whereby the only sources are the voltage sources at the $n_{p}$-port nodes, $\mathbf{B}=\mathbf{L}$ where $\mathbf{B} \in \Re^{n_{u} \times n_{p}}$. Each delayed entry of matrices $\mathbf{C}(\boldsymbol{\tau})$ and $\mathbf{G}(\boldsymbol{\tau})$ acts as a delay operator for the corresponding entry of vector $\mathbf{x}(t)$. Hence, (14) can be re-written in the Laplace domain as:

$$
\begin{aligned}
s \mathbf{C}(s) \mathbf{X}(s) & =-\mathbf{G}(s) \mathbf{X}(s)+\mathbf{B} \mathbf{V}_{p}(s) \\
\mathbf{I}_{p}(s) & =\mathbf{B}^{T} \mathbf{X}(s) \\
\mathbf{C}(s) & =\mathbf{C}_{0}+\sum_{k=1}^{n_{\tau}} \mathbf{C}_{k} e^{-s \tau_{k}} \\
\mathbf{G}(s) & =\mathbf{G}_{0}+\sum_{k=1}^{n_{\tau}} \mathbf{G}_{k} e^{-s \tau_{k}}
\end{aligned}
$$

The solution of (14) can be carried out using direct or iterative solvers. Among the direct methods, the LU decomposition has been widely used [15]. If the transient analysis is carried out for $n_{t}$ time steps, the complexity of the direct solution scales 
as $O\left(n_{t} n_{u}^{3}\right)$, where $n_{u}$ is the total number of unknowns. Hence, for a large number of unknowns $n_{u}$, equations (14) cannot be solved directly and iterative solvers are needed. Assuming that an average number $n_{\text {iter }}$ of iterations per time step is required for the iterative solver to converge to a specified residual and $n_{u}^{2}$ is the computational cost for the matrix-vector products involved, the iterative solution for all time steps $n_{t}$ scales as $O\left(n_{\text {iter }} n_{t} n_{u}^{2}\right)$ [16].

\section{Conclusions}

We have presented an overview of the PEEC method in the solution of the EFIE with special focus on the analysis of electrically long structures that require taking delays into account. When signal waveform rise times decrease and the corresponding frequency content increases or the geometric dimensions become electrically long, time delays must be taken into account and included in the modeling.

\section{Acknowledgments}

This work was supported by the Research Foundation Flanders (FWO).

\section{References}

[1] R. Achar, M. Nakhla, "Simulation of highspeed interconnects," Proceedings of the IEEE, vol. 89, no. 5, pp. 693-728, May 2001.

[2] A. E. Ruehli and A. C. Cangellaris, "Progress in the methodologies for the electrical modeling of interconnects and electronic packages," Proc. IEEE, vol. 89, no. 5, pp. 740-771, May 2001.

[3] R. F. Harrington, Field Computation by Moment Methods. New York: Macmillan, 1968.

[4] A. E. Ruehli, "Equivalent circuit models for three dimensional multiconductor systems," IEEE Trans. Microw. Theory Tech., vol. 22, no. 3, pp. 216-221, Mar. 1974.

[5] J. M. Jin, The Finite Element Method in Electromagnetics, 2nd ed. John Wiley and Sons, New York, 2002.

[6] C. A. Balanis, Advanced Engineering Electromagnetics. John Wiley and Sons, New York, 1989.

[7] W. Pinello, A. C. Cangellaris, and A. Ruehli, "Hybrid electromagnetic modeling of noise interactions in packaged electronics based on the partial-element equivalent-circuit formulation," IEEE Trans. Microw. Theory Tech., vol. 45, no. 10, pp. 1889-1896, Oct. 1997.

[8] L. W. Nagel, "SPICE: A computer program to simulate semiconductor circuits," University of California, Berkeley, Electr. Res. Lab. Report ERL M520, May 1975.

[9] P. J. Restle, A. Ruehli, S. G. Walker, G. Papadopoulos, "Full-wave PEEC time-domain for the modeling of on-chip interconnects," IEEE Transactions on Computer-Aided Design, vol. 20, no. 7, pp. 877-887, July 2001.

[10] A. E. Ruehli, G. Antonini, J. Esch, J. Ekman, A. Mayo and A. Orlandi, "Non-orthogonal PEEC formulation for time and frequency domain EM and circuit modeling," IEEE Trans. Electromagn. Compat., vol. 45, no. 2, pp. 167176, May 2003.

[11] G. Antonini, J. Ekman, "On characterizing artifacts observed in PEEC based modeling," in Proc. of the IEEE Int. Symp. on Electromagnetic Compatibility, Santa Clara, Ca, USA, Aug. 2004.

[12] J. J. H. Wang, Generalized Moment Method in Electromagnetics. John Wiley and Sons, New York, 1991.

[13] A. E. Ruehli and H. Heeb, "Circuit models for three-dimensional geometries including dielectrics," IEEE Trans. Microw. Theory Tech., vol. 40, no. 7, pp. 1507-1516, Jul. 1992.

[14] C. Ho, A. Ruehli, P. Brennan, "The modified nodal approach to network analysis," IEEE Trans. Circuits Syst., vol. 22, no. 6, pp. 504509, Jun. 1975.

[15] E. Anderson, Z. Bai, C. Bischof, S. Blackford, J. Demmel, J. Dongarra, J. Du Croz, A. Greenbaum, S. Hammarling, A. McKenney, and D. Sorensen, LAPACK Users' Guide, 3rd ed. Philadelphia, PA: Society for Industrial and Applied Mathematics, 1999.

[16] W. C. Chew, J.-M. Jin, E. Michielssen, J. Song, Fast and Efficient Algorithms in Computational Electromagnetics, A. House, Ed., 2001. 